\title{
Chronic administration of Eucommia leaf extract (ELE) and asperuloside(ASP), the major component of ELE, prevents adipocyte hypertrophy in white adipose tissues
}

\author{
Shouhei Miyazaki ${ }^{1}$, Hirotaka Oikawa ${ }^{1}$, Tetsuya Hirata ${ }^{2}$, Taro Ueda ${ }^{2}$, Wenping Zhang ${ }^{3}$, Sansei Nishibe ${ }^{4}$ and Takahiko Fujikawa ${ }^{1,5 *}$ \\ ${ }^{1}$ Laboratory of Molecular Prophylaxis and Pharmacology, Faculty of Pharmaceutical Sciences, Suzuka University of Medical Science, 3500-3 Suzuka, Minamitamagaki- \\ cho, Suzuka, Mie 513-8670, Japan \\ ${ }^{2}$ R\&D Center, Kobayashi Pharmaceutical Co., Ltd. 1-30-3, Toyokawa, Ibaraki, Osaka 567-0057, Japan \\ ${ }^{3}$ Faculty of Acupuncture \& Moxibustion, Suzuka University of Medical Science, Mie, Japan \\ ${ }^{4}$ Faculty of Pharmaceutical Sciences, Health Sciences University of Hokkaido, 1757 Kanazawa, Tobetsu-cho, Ishikari, Hokkaido 061-0293, Japan \\ ${ }^{5}$ Department of Biochemistry and Proteomics, Mie University Graduate School of Medicine, 2-174 Edobashi, Tsu, Mie 514-8507, Japan
}

\begin{abstract}
Obesity is known to be a risk factor for life style-related diseases like cardiovascular diseases, hypertension, kidney diseases and so on. Preventing obesity is beneficial for health problems and economic problems. ELE and asperuloside (ASP) have been reported to have the anti-obesity effects. In the previous study, ELE and ASP significantly reduced the ratio of WAT weight to the body weight in perirenal WAT and tended to reduce this ratio in epididymal WAT. In this study, we observed the size of the stained adipocyte in visceral WAT (perirenal and epididymal) with a microscope. ELE and ASP markedly decreased the size of adipocytes in perirenal WAT. ELE slightly decreased the size of adipocytes in epididymal WAT. ASP $(0.1,0.3 \%)$ markedly and concentration-dependent decreased in the size of adipocytes in epididymal WAT. ELE and ASP could be beneficial for curbing metabolic disorders, as they prevent the harmful effects, which are caused by the hypertrophy of adipocytes in the visceral WAT.
\end{abstract}

\section{Introduction}

Obesity is known to be a risk factor for life style-related diseases such as cardiovascular disease, diabetes, musculoskeletal disorders (especially osteoarthritis), hypertension, kidney disease, gout, and so on. Preventing obesity has a beneficial influence on not only health problems, but also economic problems. Obesity is characterized as hypertrophy and proliferation of adipocytes in white adipose tissue (WAT) $[1,2]$. Eucommia leaf extract (ELE) has been reported to have various bioactive properties [3-11], such as anti-obesity effects [611]. Asperuloside (ASP) is indicated as the major ingredient of ELE; it contributes to its anti-obesity effect $[8,11]$. In the previous study, the administration of ELE or ASP significantly reduced the ratio of WAT weight to the body weight in perirenal WAT and tended to reduce this ratio in epididymal WAT $[9,11]$. The aim of this study is to ascertain whether ELE and its major component, ASP, affect the size of adipocytes in WAT.

\section{Materials and methods}

\section{Animals and diets}

Male Sprague-Dawley rats (4 weeks old; 75-80 g), purchased from SLC, Inc., were maintained at a temperature of $23-26^{\circ} \mathrm{C}$ and a relative humidity of $50-65 \%$ for 2 weeks after they were procured. The rats were divided into four groups (Control-HFD, ELE5\%-HFD, ASP0.1\%-HFD, and ASP0.3\%-HFD; $\mathrm{n}=6$ for each group) based on body weight. Under HFD $(2635.9 \mathrm{~kJ}(630 \mathrm{kcal}) / 100 \mathrm{~g})$ conditions, all animals were fed a commercial diet containing $29.7 \%$ lard (Oriental Yeast Co. Ltd.), water
(7.7\%), protein (23.6\%), lipid (35.0\%), ash (6.1\%), dietary fiber $(2.9 \%)$, and nitrogen-free extract (24.7\%). Test foods were prepared by adding $5 \%$ ELE and 0.1 and $0.3 \%$ ASP, or by adding $5 \%$ casein as a control. The test foods were provided ad libitum for 3 months. The doses given to HFD fed animals were determined based on previous studies $[9,11]$. After chronic administration of ELE and ASP, the body weight was measured, and the rats were sacrificed without stress; the WATs (perirenal WAT and epididymal WAT) were immediately removed. The Institutional Animal Care and Use Committees at Mie University

Correspondence to: T Fujikawa, Laboratory of Molecular Prophylaxis and Pharmacology, Faculty of Pharmaceutical Sciences, Suzuka University of Medical Science, Minamitamagaki-cho, Suzuka, Mie 513-8670, Japan. e-mail: fujikawa@suzuka-u.ac.jp

Key words: Anti-obesity, asperuloside, Eucommia leaf extract, hypertrophy, white adipose tissue

Special Issue: Application to prophylactic pharmacology of food

Hirotaka Oikawa

Assistant professor

Department of Pharmaceutical Sciences

Suzuka University of Medical Science

Japan

Takahiko Fujikawa

Professor

Suzuka University of Medical Science

Japan

Received: April 27, 2018; Accepted: April 29, 2018; Published: April 30, 2018 
Miyazaki S (2018) Chronic administration of Eucommia leaf extract (ELE) and asperuloside(ASP), the major component of ELE, prevents adipocyte hypertrophy in white adipose tissues

Faculty of Medicine and the Suzuka University of Medical Science approved the animal facilities and study protocols. All procedures were performed in accordance with the National Institutes of Health guidelines for animal care.

\section{Morphological observations}

The WAT was fixed with formalin and stained with hematoxylin and eosin, as reported previously [8]. We then observed the size of the stained adipocyte in visceral WAT (perirenal and epididymal) with a microscope.

\section{Results}

Compared with the Control-HFD group, the size of adipocytes in perirenal WAT decreased markedly in 3 months in the ELE5\%-HFD group. This decrease was also observed in the ASP $(0.1,0.3 \%)$-HFD group, compared to the Control-HFD group (Figure 1). However, a dose-dependent decrease was not observed in the ASP $(0.1 \%-0.3 \%)$ HFD group. On the other hand, the size of adipocytes in epididymal WAT decreased slightly in 3 months in the ELE5\%-HFD group, compared to the Control-HFD group (Figure 2). The ASP $(0.1,0.3 \%$ )HFD group showed a marked decrease in the size of adipocytes in epididymal WAT, and a dose-dependent decrease was observed in the ASP $(0.1 \%-0.3 \%)$-HFD group (Figure 2 ).

\section{Discussion}

In the previous study, ELE and its major component, ASP, were

\section{a) Cont-HFD}

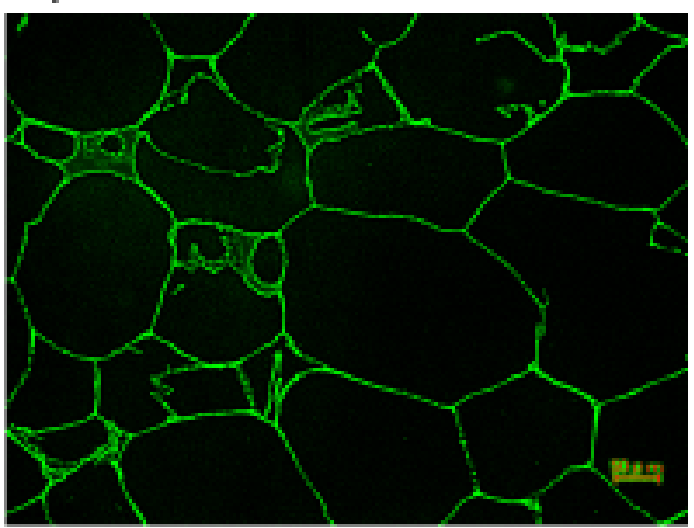

c) ASP0.1\%-HFD

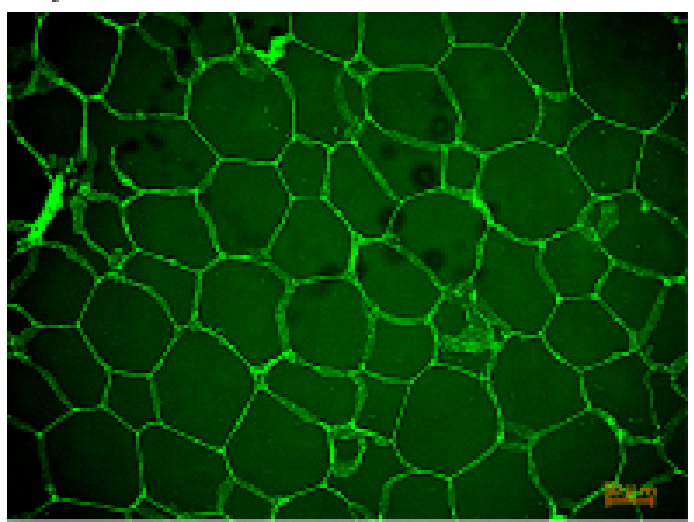

reported to result in the reduction of epididymal and perirenal WAT $[9,11]$. In this study, we found that ELE and ASP inhibited the HFDinduced hypertrophy of adipocytes in both perirenal and epididymal WAT. When WAT expansion occurs with obesity, the size, and/or the number of adipocytes is known to increase [2]. In such conditions, both ELE and ASP seemed to prevent obesity through the prevention of the hypertrophy of adipocytes in the visceral WAT. The hypertrophy of adipocytes in the visceral WAT is correlated with a high incidence of metabolic disorders. The hypertrophy of adipocytes in the visceral WAT increased basal fatty acid release, pro-inflammatory cytokine release, immune cell recruitment, hypoxia, fibrosis, decreased adiponectin, and impaired insulin sensitivity [1]. ELE and ASP could be beneficial for curbing metabolic disorders, as they prevent these harmful effects, which are caused by the hypertrophy of adipocytes in the visceral WAT.

\section{Acknowledgments}

The authors are grateful to M. Nakagawa for her technical assistance and help with statistical analysis and S. Nakao for her assistance with animal care. The Eucommia leaf extract and ASP samples used in this study were provided by KOBAYASHI Pharmaceutical Co., Ltd. (Osaka, Japan).

\section{Conflict of interest}

There are no conflicts of interest to declare.

\section{b) ELE5 $\%$-HFD}

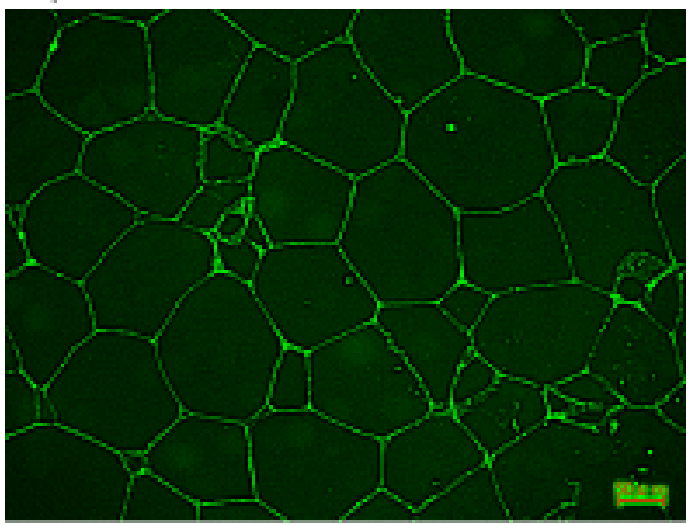

\section{d) ASP0.3\%-HFD}

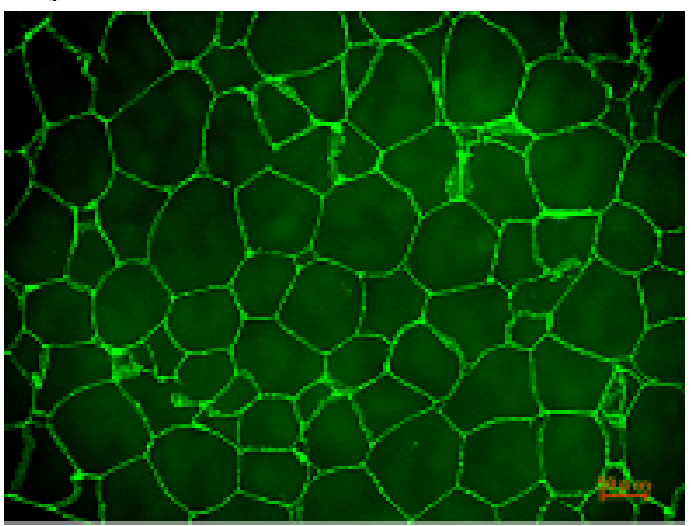

Figure 1. ELE and ASP prevented the HFD-induced hypertrophy of adipocytes in perirenal WAT. The scale bar indicates $50 \mu \mathrm{m}$. a) Control-HFD group, b) ELE5\%-HFD group, c) ASP0.1\%HFD group, d) ASP0.3\%-HFD group. 
Miyazaki S (2018) Chronic administration of Eucommia leaf extract (ELE) and asperuloside(ASP), the major component of ELE, prevents adipocyte hypertrophy in white adipose tissues

a) Cont-HFD

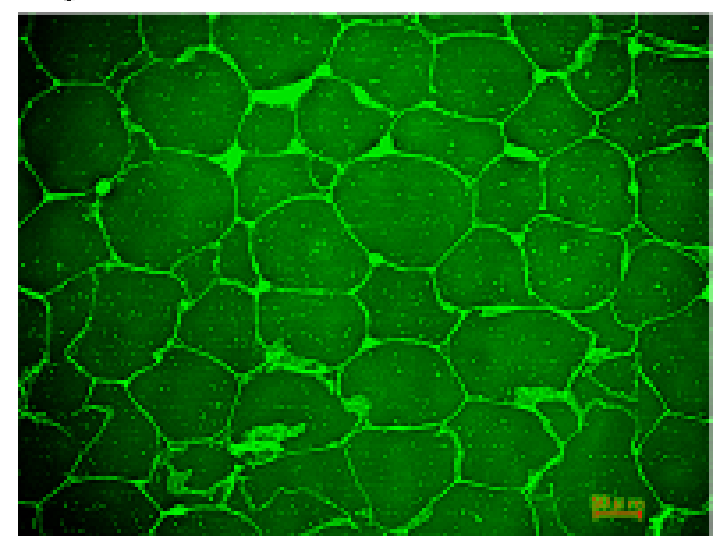

c) ASP0.1\%-HFD

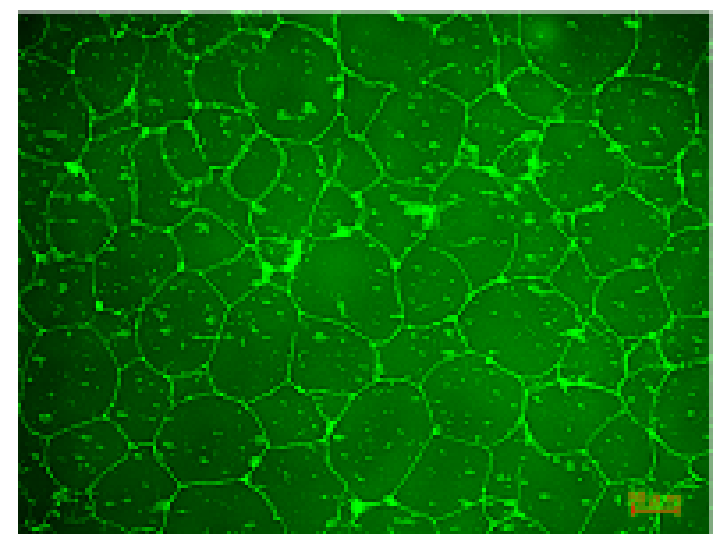

\section{b) ELE5\%-HFD}

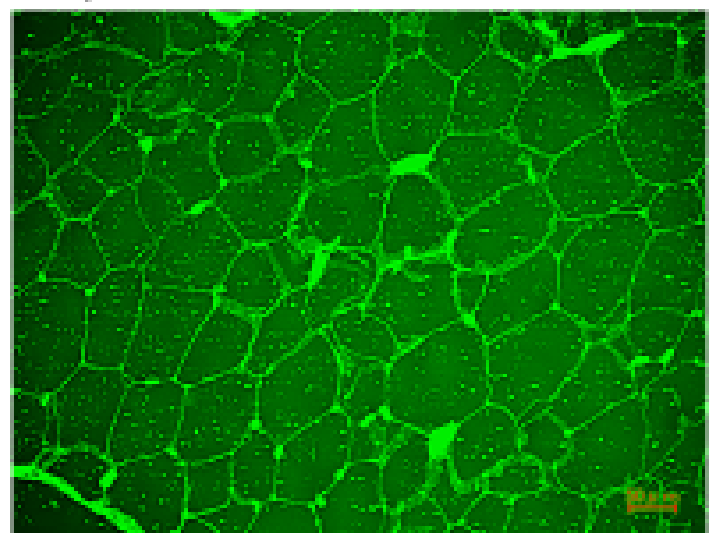

d) ASP0.3\%-HFD

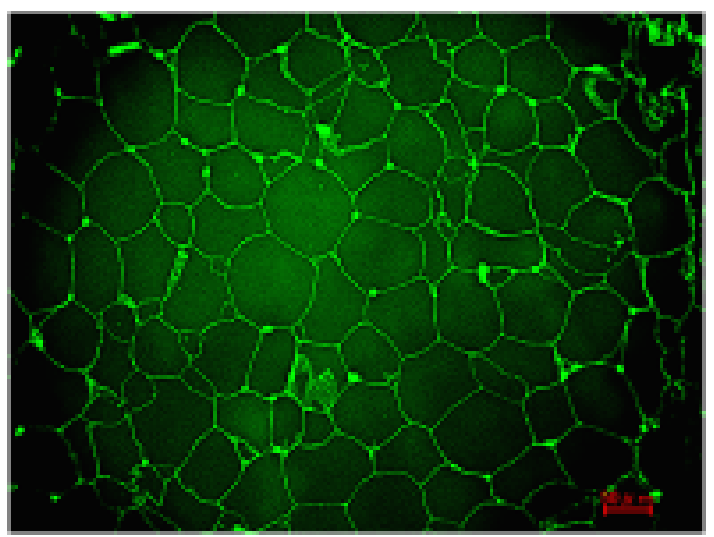

Figure 2. ELE and ASP prevented the HFD-induced hypertrophy of adipocytes in epididymal WAT. The scale bar indicates $50 \mu \mathrm{m}$. a) Control-HFD group, b) ELE5\%-HFD group, c) ASP0.1\%-HFD group, d) ASP0.3\%-HFD group.

\section{References}

1. Choe SS, Huh JY, Hwang IJ, Kim JI, Kim JB (2016) Adipose Tissue Remodeling: Its Role in Energy Metabolism and Metabolic Disorders. Front Endocrinol (Lausanne) 7: 30. [Crossref]

2. Aoki S, Sugihara H, Eguchi Y, Watanabe K (2006) The behavior of adipocytes in obesity. Nihon Rinsho 64: 175-179. [Crossref]

3. Miyazaki S, Oikawa H, Nakamichi S, Hirata T, Yamasaki H, et al. (2017) Aroma of Eucommia leaf extract (ELE) causes reduced locomotor activity and increased NREM sleep, acting like the partially related factors of oral ELE's effects with locomotoractivity-dependent-increase in NREM- and REM-sleep. Glob Drug Therap 3:2.

4. Oikawa H, Miyazaki S, Nishida K, Zhang W, Hirata T, et al. (2017) Promotion of osteoblastic $\mathrm{Ca}^{2+}$ accumulation by Eucommia leaf extract. Glob Drug Therap 3:2

5. Hiramoto K, Goto K, Sekijima H, Ooi K, Hirata T, et al. (2017) The preventive effects of asperuloside administration on dextran sodium sulfate-induced ulcerative colitis in mice. Glob Drug Therap 3:2.

6. Oikawa H, Miyazaki S, Zhang W, Nishide H, Nakamichi S, et al. (2017) The characteristic taste of Eucommia leaf extract as the additive of a weight-loss and the constipation improvement is not associated with suppression of the feeding behavior of the fast rats with the extract. Glob Drug Therap 3:2.

7. Oikawa H, Nakamich S, Nishide H, Kawaguchi N, Zhang W, et al. (2016) Engagement of the aroma in feeding suppression behavior of Eucommia leaf. J Aroma Sci Technol Safety 17: 74-79.

8. Hirata T, Kobayashi T, Wada A, Ueda T, Fujikawa T, et al. (2011) Anti-obesity compounds in green leaves of Eucommia ulmoides. Bioorg Med Chem Lett 21: 17861791. [Crossref]
9. Fujikawa T, Hirata T, Wada A, Kawamura N, Yamaguchi Y, et al. (2010) Chronic administration of Eucommia leaf stimulates metabolic function of rats across several organs. Br J Nutr 104: 1868-1877. [Crossref]

10. Zhang W, Fujikawa T, Mizuno K, Ishida T, Ooi K, et al. (2012) A Eucommia leaf extract (ELE) prevents OVX-induced osteoporosis and obesity in rats. Am J Chin Med 40: 735-752. [Crossref]

11. Fujikawa T, Hirata T, Hosoo S, Nakajima K, Wada A, et al. (2012) Asperuloside stimulates metabolic function in rats across several organs under high-fat diet conditions, acting like the major ingredient of Eucommia leaves with anti-obesity activity. J Nutr Sci 1: e10. [Crossref]

Copyright: (C2018 Miyazaki S. This is an open-access article distributed under the terms of the Creative Commons Attribution License, which permits unrestricted use, distribution, and reproduction in any medium, provided the original author and source are credited. 